\title{
Optical coherence tomography with a Fizeau interferometer configuration
}

\author{
P. Casaubieilh, H. D. Ford, S. W. James and R. P. Tatam \\ Optical Sensors Group, Centre for Photonics and Optical Engineering, School of Engineering, \\ Cranfield University, Bedfordshire, MK43 0AL, UK
}

\begin{abstract}
We report the investigation of a Fizeau interferometer-based OCT system. A secondary processing interferometer is necessary in this configuration, to compensate the optical path difference formed in the Fizeau interferometer between the end of the fibre and the sample. The Fizeau configuration has the advantage of 'downlead insensitivity', which eliminates polarisation fading. An optical circulator is used in our system to route light efficiently from the source to the sample, and backscattered light from the sample and the fibre end through to the Mach-Zehnder processing interferometer. The choice of a Mach-Zehnder processing interferometer, from which both antiphase outputs are available, facilitates the incorporation of balanced detection, which often results in a large improvement in the Signal-toNoise ratio (SNR) compared with the use of a single detector. Balanced detection comprises subtraction of the two antiphase interferometer outputs, implying that the signal amplitude is doubled and the noise is well reduced.

It has been discerned that the SNR drops when the refractive index variation at a boundary is small. Several OCT images of samples (resin, resin + crystals, fibre composite) are presented.
\end{abstract}

Key-words: Optical Coherence Tomography, Fizeau interferometer, optical fibres

\section{INTRODUCTION}

Optical Coherence Tomography (OCT) is an imaging technique analogous to ultrasound (US) but instead of using sound, backscattered light from the sample is detected, providing a resolution higher than that obtained from US. OCT has been applied to the imaging of biological tissue but has also been applied to depth imaging in composite materials ${ }^{1}$. This technique generally uses low-coherence interferometry employing an interferometer in which interference fringes are observed when the optical path difference between the reference and sample arms is within the coherence length of the source.

Typically the optical imaging system is configured using an in-fibre Michelson interferometer. However this arrangement has to be carefully configured to avoid different polarisation state changes between the fibre arms leading to visibility fluctuations, dispersion due to the different types of fibre used and phase noise. In order to minimise environmental sensitivity, we have employed a Fizeau interferometer configuration (figure 1). 


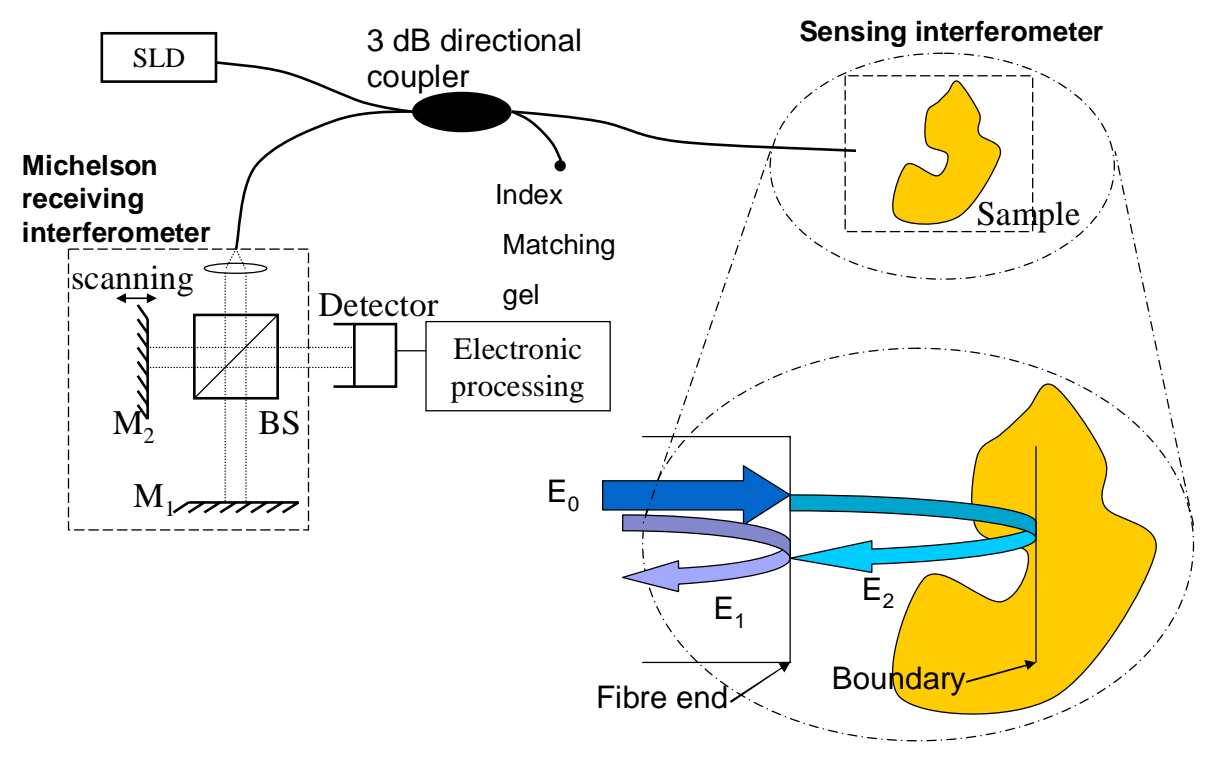

Figure 1: Basic Fizeau configuration, with Michelson processing interferometer

The light from a broadband source passes through the $3 \mathrm{~dB}$ fibre coupler and is transmitted to the sensing interferometer where two reflections occur: one part of the light is reflected at the end of the fibre (the reference arm) and the other part is reflected, transmitted and scattered by the sample at the different internal boundaries (the sample arm). Both the reflection at the end of the fibre and the backscattered light pass through the same fibre to the processing interferometer where each component suffers amplitude splitting into two parts and reflection at both mirrors. Four components of electric fields reach the detector and a maximum of $25 \%$ of the initial power is available.

The performance of the system is defined by the signal-to-noise ratio (SNR), which determines how well the system is able to differentiate the refractive index change at the boundaries. The maximum SNR is defined as the square of the maximum intensity of the fringes obtained for a mirror in the sample arm divided by the variance of the noise when the sample arm is blocked.

In this paper, the latest improvements of the Fizeau OCT system arrangement employing balanced detection are presented. The sensitivity of the system to differentiate small refractive index changes is discussed. 


\section{THEORY}

\subsection{Fizeau Interferometer configuration}

The basic configuration is shown in figure 1.

The advantages of this configuration compared to the Michelson interferometer, and possible improvements have been discussed previously ${ }^{2,3}$. In this system interference occurs when the optical path difference in the processing interferometer is equal to the optical path in the sensing interferometer, that is when the optical path difference between the processing and sensing interferometers is within the coherence length of the source.

The SNR of this configuration can be improved by incorporating an optical circulator instead of a $3 \mathrm{~dB}$ directional coupler and implementing balanced detection. Several system topologies are available to implement balanced detection but an optimum design that employs a Mach-Zehnder processing interferometer is shown in figure $2^{2}$.

\subsection{Signal-To-Noise Ratio (SNR)}

The received optical power in an OCT system is usually low. Broadband, infra-red solid-state sources are generally used because they allow good tissue penetration and depth resolution, and reflectivity values at tissue interfaces are small. Thus optical power levels at the detector fall typically in the nanowatt to microwatt range. Ideally, systems are operated at high bandwidth to enable fast scanning and rapid acquisition of images. The combination of low power and high bandwidth means that the signal-to-noise ratio (SNR) in OCT is likely to be relatively low for standard detection techniques, and that careful consideration will have to be given to system design. The value of the SNR must exceed that required to resolve the small refractive index variations (around 0.01-0.1) typical of biological tissue ${ }^{4}$.

The maximum SNR of an OCT system ${ }^{5}$ is defined as the ratio of the mean square signal photocurrent $\left\langle I_{s}{ }^{2}\right\rangle$ from the detector, under path-matched conditions, to the total photocurrent variance $\sigma_{i}{ }^{2}$.It is given by:

$$
\mathrm{SNR}=10 \log _{10}\left(\frac{\left\langle\mathrm{I}_{\mathrm{s}}^{2}\right\rangle}{\sigma_{\mathrm{i}}^{2}}\right)
$$

The SNR calculation makes use of the optical power $P_{r}$ in the reference beam, power $P_{s}$ in the sample beam and the optical power $P_{x}$ of stray incoherent light at the detector. Corresponding power reflectivities $R_{r}, R_{s}$ and $R_{x}$ are also used and the responsivity $\rho$ of the detector is required. For maximum fringe visibility, the states of polarisation of the interfering beams must be identical, or reductions in SNR will occur. This paper assumes unpolarised light from the source. Although this is not always the case experimentally, it is a valid assumption for the purpose of comparing the maximum expected SNR for a range of proposed interferometer configurations. $\left\langle\mathrm{I}_{\mathrm{s}}^{2}\right\rangle$ is a function of the optical powers in the reference and signal beams and of the detector responsitivity.

There are three sources of noise to be considered in calculating the SNR of any OCT system. The first is receiver noise, which arises due to the random thermal motion of electrons within the the PIN photodetector. As it is followed by an electronic circuit in order to amplify the signal, a receiver noise component due to the amplification has normally to be taken in account. For commercial photodetectors, the receiver noise is usually specified by the manufacturer (in terms of NEP (Noise Equivalent Power $(\mathrm{W} / \sqrt{\mathrm{Hz}})$ )), it is usually expressed by ${ }^{6,7}$ :

$$
\sigma_{\mathrm{re}}^{2}=\rho(\mathrm{NEP})^{2} \mathrm{~B}
$$

where $B$ is the detection bandwidth ${ }^{8}$. The two other sources of noise are both dependent upon the average photocurrent $I_{d c}$ at the detector or, in the case of balanced detection, detectors. Shot noise arises as a result of the random distribution in 
arrival times of photons at the detector from a monochromatic light source. The shot noise photocurrent variance $\sigma_{s h}{ }^{2}$ that results from this Poisson process is given by:

$$
\sigma_{\mathrm{sh}}^{2}=2 \mathrm{qI}_{\mathrm{dc}} \mathrm{B}
$$

where $q$ is the electronic charge.

The final source of noise to be considered is excess photon noise, which arises due to the random arrival of photons from a broad band light source. This is a Bose-Einstein process, with different statistics from shot noise, and the photocurrent variance for the excess noise is given by

$$
\sigma_{\mathrm{ex}}^{2}=\mathrm{I}_{\mathrm{dc}}^{2} \frac{\mathrm{B}}{\Delta v}
$$

Here, $\Delta v$ is the frequency linewidth of the source.

When a balanced receiver can be used, much of the excess noise has identical time dependence in both detectors, and is therefore cancelled. The dominant remaining noise contribution at moderate optical powers is often shot noise, and the resulting improvement in SNR can be as much as $40 \mathrm{~dB}$. However, in some configurations there can be a phase difference between the beams arriving at the two detectors for stray, incoherent beams arising from unwanted reflections ${ }^{9}$. In this situation, the beating that occurs between non-coherent spectral components of the broadband source gives rise to a form of noise called beat noise. The beat noise is given by:

$$
\sigma_{\text {be }}^{2}=\rho^{2} \mathrm{P}_{\mathrm{x}} \mathrm{P}_{\mathrm{r}} \mathrm{B} / \Delta v \text {. }
$$

The beat noise may appear in the Michelson interferometer due to scattering at the end of the fibre; it is typically minimised by polishing the fibre end or using some index matching gel. For the Fizeau configuration, the reflection at the end of the fibre is used to generate the reference signal which removes the beat noise term, and it enables to neglect it.

The arrangement presented in figure 2 is used to determine the $I_{d c}$ and $I_{S}$ expressions.

\section{EXPERIMENTAL}

The experimental set-up is shown in figure 2. The light from the broadband source (a superluminescent diode centred at $1550 \mathrm{~nm}$, FWHM of $40 \mathrm{~nm}, 1 \mathrm{~mW}$ ) is directed by the optical circulator (OC) to the Fizeau sensing interferometer. Both the reflected light at the end of the fibre and the backscattered light are directed to the MachZehnder processing interferometer. The light is divided into two parts that will recombine at the second beam splitter. The transmitted light is reflected by the corner cube and the mirror and the beams recombine at the second beam splitter.

A balanced photoreceiver from New Focus (Model: 2017, NEP=3pW/ $\sqrt{\mathrm{Hz}}, \mathrm{B}=125 \mathrm{kHz}$ ) receives the two complementary outputs of the Mach-Zehnder interferometer, enabling doubling of the amplitude of the signal and subtraction of the noise. The signal is acquired by an A/D card and displayed on a PC. The corner cube is mounted on a translation stage (speed: $0.3 \mathrm{mms}^{-1}$ ) which is controlled by means of a Labview ${ }^{\mathrm{TM}}$ program. 


\subsection{Expressions for SNR investigation}

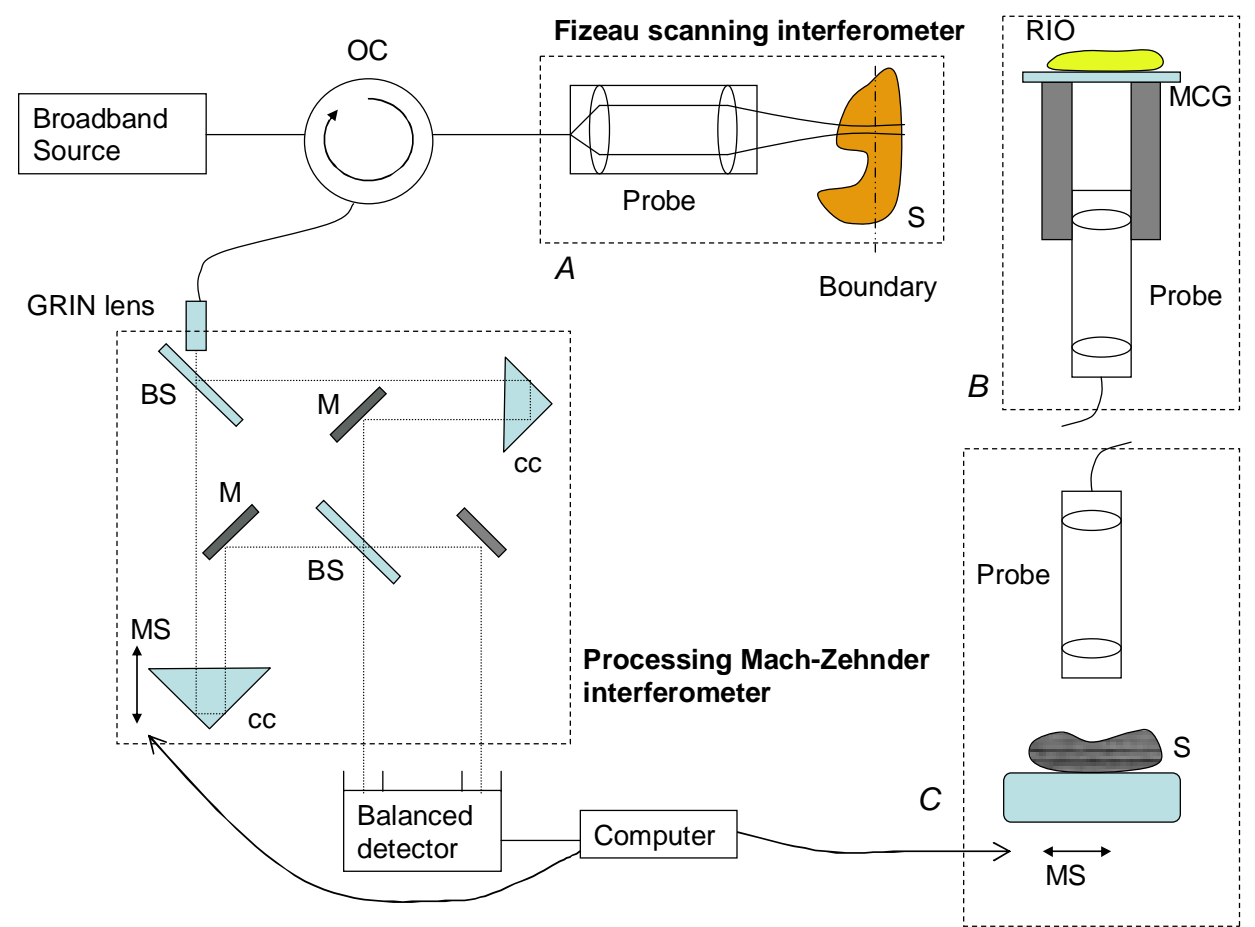

Figure 2: Balanced Fizeau configuration (A) with the sample (B); OC: optical circulator, BS: beamsplitter, M: mirror, cc: corner cube, MV: moving stage, S: sample, MCG: microscope cover glass; RIO: refractive index oil; D1, D2: detectors

Using 50/50 beamsplitters and mirrors (figure 2), it can be shown that the reference light power $\mathrm{P}_{\mathrm{r}}$ and the sample light power $\mathrm{P}_{\mathrm{s}}$ detected by the detector are expressed by:

$$
\begin{aligned}
& \mathrm{P}_{\mathrm{r}}=\frac{1}{4}\left(\mathrm{P}_{0} \mathrm{~T}_{\mathrm{c}}^{2} \mathrm{R}_{\mathrm{e}}\right) \\
& \mathrm{P}_{\mathrm{s}}=\frac{1}{4}\left(\mathrm{P}_{0} \mathrm{~T}_{\mathrm{c}}^{2}(1-\mathrm{Re})\left(1-\mathrm{R}_{\mathrm{ag}}\right) \mathrm{R}_{\mathrm{s}}\right)
\end{aligned}
$$

Where $P_{0}$ is the initial power, $T_{c}$ is the transmission ratio of the optical circulator, $R_{e}$ is the reflectivity at the end of the fibre, $R_{a g}$ is the reflectivity at the air-glass interface, and $R_{s}$ is the reflectivity at the boundary of interest: the glass-oil interface (it has to be noted that in the rest of the calculations the reflection at oil-air interface is neglected).

The intensity of the interference peak at the boundary is given by:

$$
\mathrm{I}_{\mathrm{S}}=\frac{1}{2} \rho \mathrm{P}_{0} \mathrm{~T}_{\mathrm{c}}^{2} \sqrt{\left(1-\mathrm{R}_{\mathrm{e}}\right)\left(1-\mathrm{R}_{\mathrm{ag}}\right) \mathrm{R}_{\mathrm{S}}}
$$

And as well the dc photocurrent intensity $\mathrm{I}_{\mathrm{dc}}$ is given by:

$$
\mathrm{I}_{\mathrm{dc}}=\frac{1}{2} \mathrm{P}_{0} \mathrm{~T}_{\mathrm{c}}^{2}\left[\mathrm{R}_{\mathrm{e}}+\left(1-\mathrm{R}_{\mathrm{e}}\right)\left(1-\mathrm{R}_{\mathrm{ag}}\right) \mathrm{R}_{\mathrm{s}}\right]
$$

In the Fizeau arrangement, beat noise can be neglected, because the reflection at the end of the fibre acts as the reference rather than a superfluous reflection. When balanced detection is used, the total noise $\sigma_{i}^{2}$ in the system is given by: 


$$
\sigma_{i}^{2}=2\left(\sigma_{\mathrm{ex}}^{2}+\sigma_{\mathrm{sh}}^{2}\right)
$$

Replacing equations 9 and 7 in equation 1 , the $\operatorname{SNR}(\mathrm{dB})=\mathrm{f}\left(\mathrm{n}_{\text {oil }}\right)$ can be plotted

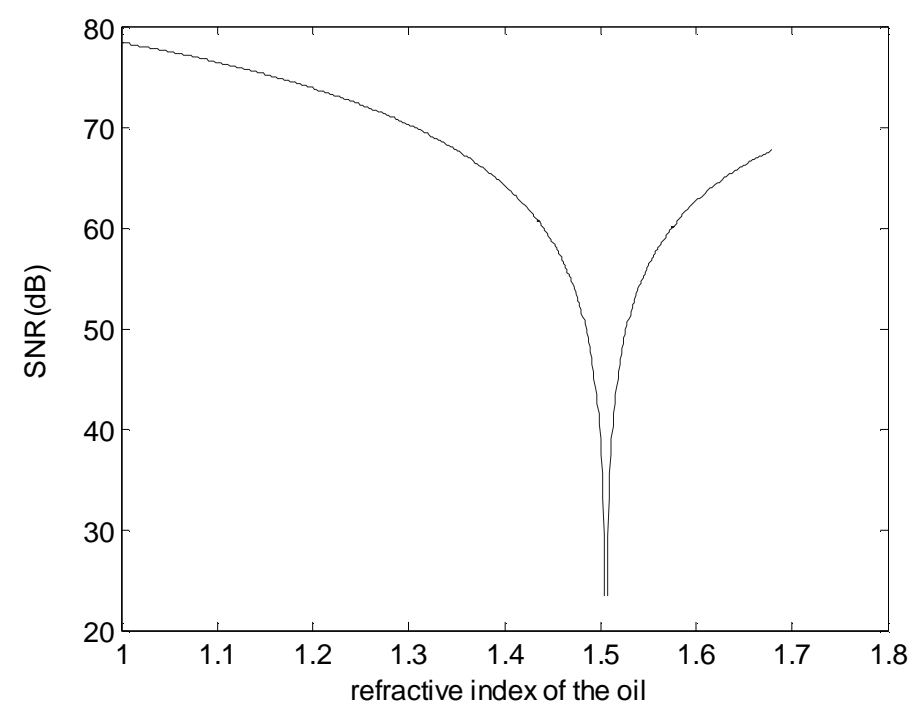

Figure 3: Theoretical SNR plot via the refractive index supposing that the substrate is a glass with a refractive index of 1.504

As expected, the reflectivity decreases as the refractive index of the oil approaches that of the glass substrate, with a corresponding decrease in SNR.

As detailed below, experiments have been performed in order to compare the theoretical expression with experimental results obtained under controlled conditions.

\section{RESULTS}

In order to investigate the efficiency of the system to differentiate small variations of refractive index, samples of refractive index oils were positioned as shown in figure 2-(B). The probe was placed vertically, and a tube enabled positioning of the sample in the Rayleigh range of the focused beam. When a longitudinal scan was carried out for a 0.2 mm-thickness glass cover slip, the same peak amplitudes were observed for both surfaces of the glass cover slip. Different refractive index oils (from Cargille Industry), from a range of 1.400 to 1.700, were deposited on the surface of the glass slide and 20 scans were carried out in order to get the average and the standard deviation of the SNR value. The sensitivity has been tested with distilled water and air as well as oils. In these measurements the refractive index of the glass was taken as reference.

The system was then applied to further samples: one sample was a droplet of resin placed on a glass slide (figure 5-(a)), and another was a cured fibre composite (figure 7-(a)).

133 longitudinal scans separated by $30 \mu \mathrm{m}$ were taken for different positions on the sample in order to produce a two-dimensional image.

Prior to carrying out an experiment with each different oil, the glass slide was cleaned with water and then isopropanol. By using different refractive index oils, the reflectivity at the glass-oil boundary is changed. The measured 
refractive index of the glass was 1.504 at $1550 \mathrm{~nm}$-wavelength and it is expected that the SNR will fall quickly when the value of the refractive index of the oil approaches the refractive index of the glass slide.

Figure 4 shows the experimentally measured results of the SNR as a function of the change in oil refractive index.

SNR evolution with refractive index

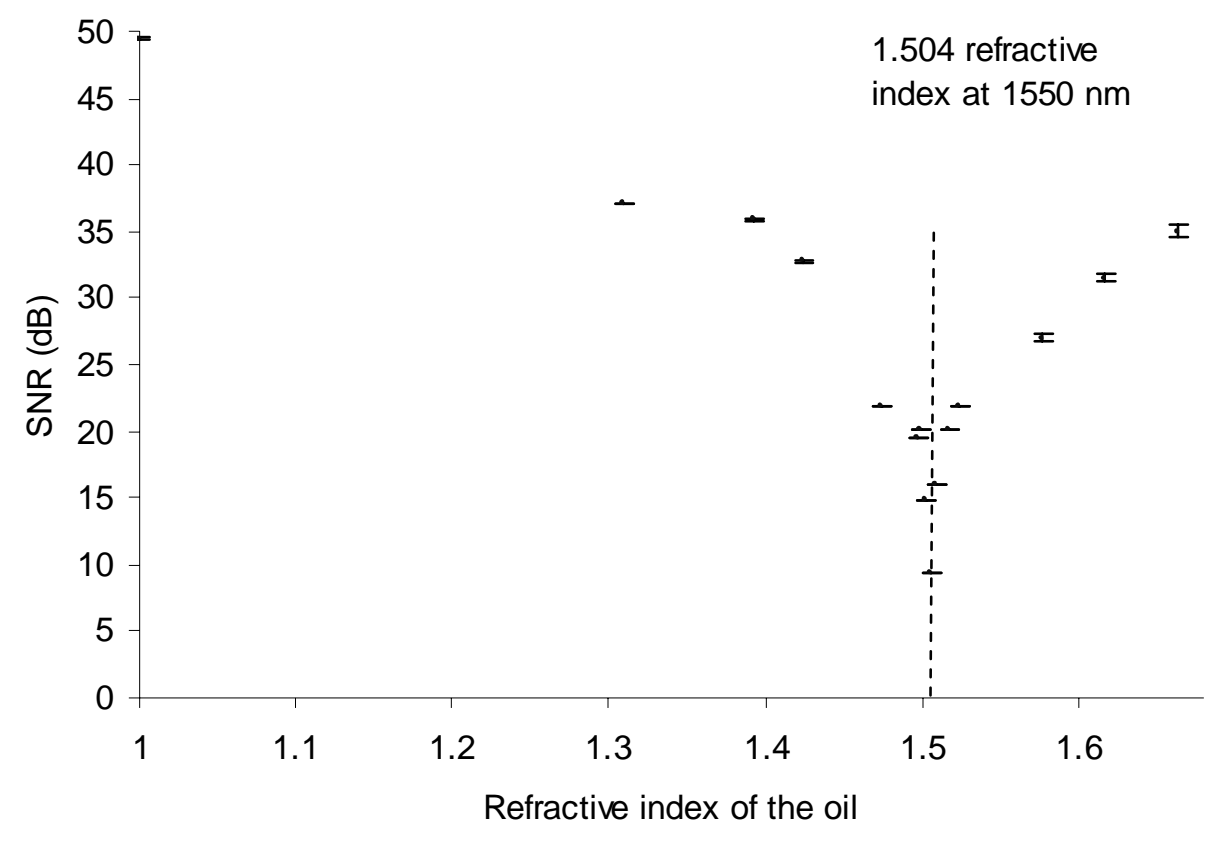

Figure 4: SNR evolution via the refractive index of the oil

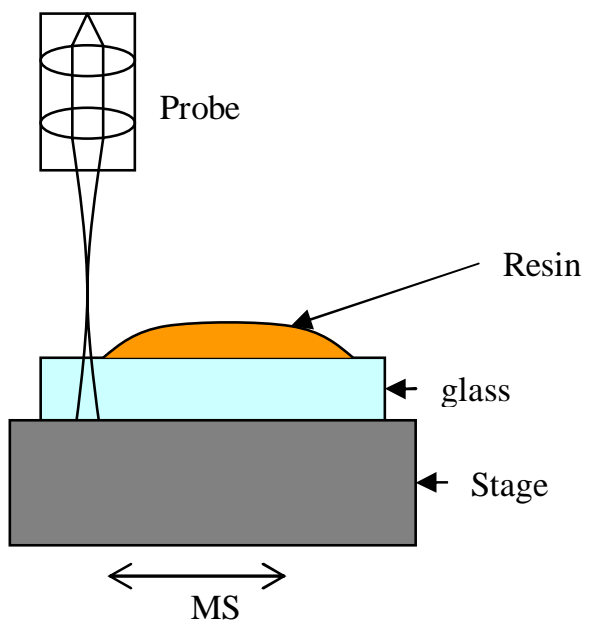

(a)

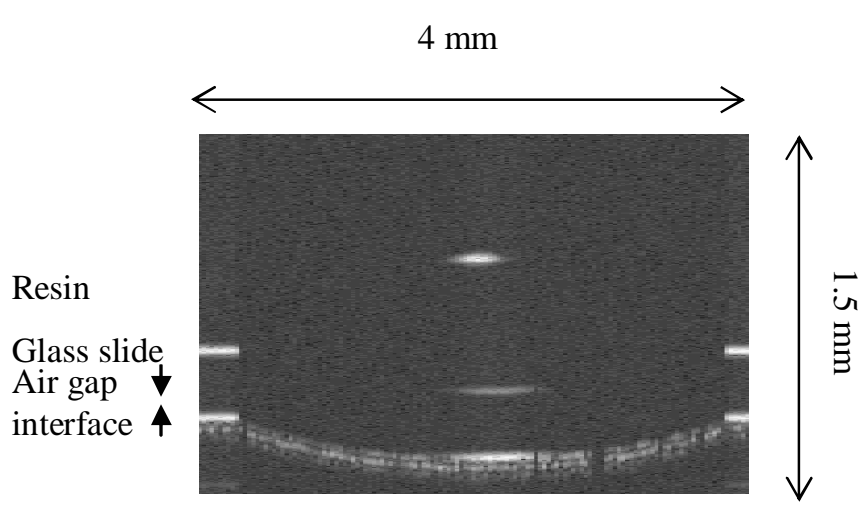

(b)

Figure 5: (a) experimental set-up of the sample under test, (b): OCT image of the droplet on the glass 


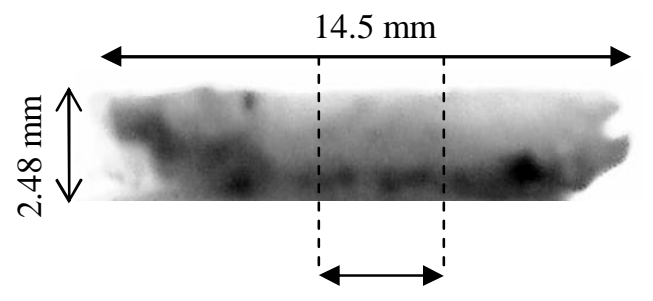

Region of observation

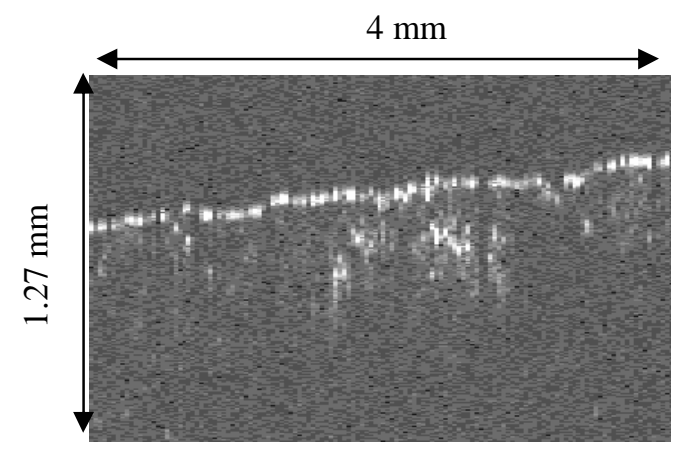

(b)

(a)

Figure 6: (a) cured fibres resin block and (b) OCT image of the region of observation

\section{DISCUSSION}

From the graph of the SNR as a function of refractive index, it is evident that the theoretical and experimental curves have the same form. It is also clear that the experimental SNR is generally much lower than the theoretical curve by about $30 \mathrm{~dB}$. This is due to a limitation from the dynamic range of the D/A card, being $30 \mathrm{~dB}$ (12 bit) and also to the fact that NEP takes into account only the noise generated in the PIN detector and not the total detector noise.

From the first sample, shown in figure 5-b, different layers are detected. The top of the resin is observed but it is not possible to distinguish the rest of the surface of the resin-air and resin-glass interface. This is because of the specular reflection from the surface of the air-resin interface; a similar remark can be done for the interface resin-glass. An air gap is observed between the glass and the translation stage (this is because the stage has a rough surface and acts as a diffuse reflector). Finally the resin droplet is thicker on the centre compared to the edge, providing a bigger optical path difference and explaining the observed distortion on the cross sectional image.

Finally, reflection sites are observed in the cured resin fibre composite block due to fibres that are not cured, air bubbles or impurities existing between the different layers before curing.

\section{CONCLUSION}

The SNR of the optimum balanced Fizeau arrangement employing an optical circulator, a processing MachZehnder interferometer and a balanced photoreceiver has been investigated. The minimum refractive index change that the system is capable of detecting has been investigated. The system has been demonstrated for transparent samples, and also for semi-transparent material including a sheet of cured resin fibre composite.

Detailed studies of system performance were carried out in order to determine the SNR of the system under typical experimental conditions. Similar SNR evolutions as a function of the refractive index are expected for other configurations. 


\section{REFERENCE}

1. Duncan, M.D. and Bashkansky, M. (1998) Surbsurface Defect Detection In Materials Using Optical Coherence Tomography. 2, 540-545.

2. Ford, H.D., Beddows, R., Casaubieilh, P., and Tatam, R.P. (2005) Comparative Signal-To-Noise Analysis Of FibreOptic Based OCT systems.

3. Casaubieilh, P., Ford, H. D., and Tatam, R. P. Optical Fibre Fizeau-Based OCT. Second European Workshop On Optical Fibre Sensors. 2004. Santander.

4. Qu, J., MacAulay, C., Lam, S., and Palcic, B. (1995) Laser Induced Fluorescence Spectroscopy At Endoscopy: Tissue Optics; Monte Carlo Modeling And In Vivo Measurements. 34, 3334-3343.

5. Rollins, A.M. and Izatt, J.A. (1999) Optimal Interferometer Designs For Optical Coherence Tomography. 24, 14841486.

6. Frosz, Michael H., Juhl, Michael, and Lang, Morten H. Optical Coherence Tomography: System Design And Noise Analysis. 2001.

7. Rollins, A. and Izatt, J.A. (2000) SNR Analysis of Conventional and Optimal Fiber-Optic Low-Coherence Interferometer Topologies. 3915, 60-67.

8. Oliver, B. M. Signal-To-Noise Ratios In Photoelectric Mixing. Proc. IRE. 49. 61.

9. Takada, K. Noise In Optical Low-Coherence Reflectometry . IEEE Journal Of Quantum Electronics. 34. 98. 